\section{Deformation mechanisms in polylactic acid/natural rubber/organoclay bionanocomposites as revealed by synchrotron X-ray scattering}

\author{
Natacha Bitinis, ${ }^{* a}$ Alejandro Sanz, ${ }^{* b c}$ Aurora Nogales, ${ }^{b}$ Raquel Verdejo, ${ }^{a}$ Miguel A. Lopez-Manchado ${ }^{a}$ \\ and Tiberio A. Ezquerra ${ }^{b}$
}

Received 29th March 2012, Accepted 27th June 2012

DOI: $10.1039 / \mathrm{c} 2 \mathrm{sm} 25729 \mathrm{~g}$

The micromechanical deformation mechanisms of a polylactic acid (PLA)/natural rubber (NR) blend (PLA/NR 90/10 wt\%) and its organoclay filled bionanocomposites have been investigated by small and wide angle X-ray scattering (SAXS-WAXS) under tensile conditions. The addition of NR to a PLA matrix changed the brittle fracture of PLA to a ductile deformation through the debonding of the rubber droplets. Otherwise, the formation of cavities between PLA and NR was hampered by the nanoclays since they were mainly located at the polymer blend interface. In this case, the nanoclays acted as craze nucleation sites. At $1 \mathrm{wt} \%$ of filler concentration, the crazes were able to fully develop in the blend and to evolve into stable microvoids, which kept growing and orienting in the tensile direction. These mechanisms also explained the progressive plastic deformation of the polymer chains and the preferential orientation of the nanoclay platelets.

\section{Introduction}

Polylactic acid (PLA) is a linear aliphatic polyester that has found plenty of applications in a broad range of medical procedures, including drug delivery systems and tissue engineering, among others. ${ }^{1-3}$ However, polylactic acid presents a high brittleness and mechanical weakness, with a very low elongation at break and low impact resistance. Being both biodegradable and biocompatible, the improvement of the mechanical properties of polylactic acid by using different procedures, including blending with other natural or biodegradable polymers or mixing with nanoadditives, appears to be of great intrinsic interest. ${ }^{4-6}$

Blending two polymers is a practical way to develop new polymeric materials that effectively combine the properties of the components. ${ }^{7,8}$ As an example, rubber matrices have commonly been used as a second polymeric phase to improve the toughness of brittle thermoplastic materials, receiving much attention from both academic and industrial areas. The properties of these blends strongly depend on the microscopic morphology which in turn determines the micromechanical deformation mechanisms, such as internal rubber cavitation, rubber droplet/matrix debonding, shear yielding or crazing, the latter being defined as microvoids bridged by small fibrils. ${ }^{9-11}$

anstituto de Ciencia y Tecnologia de Polimeros, ICTP-CSIC, C/Juan de la Cierva, 3, 28006 Madrid, Spain. E-mail: nbitinis@ictp.csic.es; Fax: +34 915644853; Tel: +34 912587561

${ }^{b}$ Instituto de Estructura de la Materia, IEM-CSIC, Serrano 121, 28006 Madrid, Spain.E-mail: alejandro.sanz@csic.es

'Instituto de Química Física Rocasolano, IQFR-CSIC, Serrano 119, 28006 Madrid, Spain
Recently, it has been demonstrated that the nanoparticles behave as effective compatibilisers for immiscible polymers through their preferential location at the polymer interfaces, reducing the interfacial tension and preventing the coalescence of the dispersed phase. ${ }^{12-16}$ Moreover, Vo and Giannelis demonstrated that such ternary blends can be stiffer, stronger and also tougher than the binary blends suggesting a change in their deformation mechanisms. ${ }^{13}$ Dasari et al. proposed an analysis of the micro- and nanoscale deformation behaviour for nylon 66/SEBS-g-MA/organoclay ternary nanocomposites using microscopy analysis of the fracture surface. ${ }^{17}$ However, microscopy analysis only provides snapshot information of the deformation mechanism with no real time monitoring and requires an extensive and laborious sample preparation after breaking. Deformation mechanisms can be investigated "in situ" by combining scattering techniques using synchrotron light with stress-strain experiments in a simultaneous fashion. ${ }^{18,19}$ This approach has already been used to study polymer blends and polymer nanocomposites since it enables linking the macroscopic deformation to the structural changes at both microscopic and mesoscopic levels. ${ }^{19-21}$ However, to the best of our knowledge, no reports regarding nanoparticle filled polymer blends are found in the literature.

In our previous study, we demonstrated the production of ductile PLA through the addition of natural rubber (NR). ${ }^{22}$ Further improvements of these PLA/NR blends were obtained by developing new bionanocomposites. Bionanocomposites can be considered as a subset of polymer nanocomposites where the nanofillers, the matrix, or both, come from bio-based, renewable resources. In our case, a small amount of organoclays was added to a bio-based polymer blend, allowing a further increase of the 
elongation at break. This effect was attributed to the preferential location of the organoclays at the PLA/NR interface. ${ }^{23}$ In this work, we seek to provide a better understanding of the micromechanical deformation mechanism of bionanocomposites based on organoclay filled PLA/NR blends during uniaxial stretching by using simultaneous synchrotron small angle and wide angle scattering (SAXS, WAXS) techniques. By performing such an analysis we aim to obtain information at different length scales relevant to the deformation mechanism. On one hand, WAXS can be used to provide information about length scales associated with inter-chain correlations of the polymer matrix. On the other hand, SAXS can provide structural information about microvoid formation, crazing, shear yielding or debonding phenomena. Moreover, additional information about the organoclay orientation can be obtained from the intermediate angle range of the scattering and from there a precise picture about the evolution with deformation of the PLA/NR interface can be inferred. The simultaneous combination of this battery of techniques with the stress-strain experiment allows us to precisely describe the deformation mechanism in this class of bionanocomposites.

\section{Experimental part}

\section{Materials and sample preparation}

PLA was provided by NatureWorks ${ }^{\circledR}$. The selected grade, PLA 2002D (D-content $4.25 \%, \mathrm{MI}=5-7 \mathrm{~g} / 10 \mathrm{~min}$ ), is a semicrystalline extrusion material with a residual monomer content of $0.3 \%$. Natural Rubber (NR) was kindly supplied by Malaysian Rubber under the trade name CV60 (Mooney viscosity ML $(1+4)$ $100{ }^{\circ} \mathrm{C}=60$ ). An organoclay (Cloisite 15A, a montmorrillonite modified with alkyl ammonium with a basal spacing of $3.15 \mathrm{~nm}$ ) purchased from Southern Clays Products was used as filler. PLA and $\mathrm{C} 15 \mathrm{~A}$ were dried overnight in a desiccating dryer at $80^{\circ} \mathrm{C}$ prior to use. The nanocomposites were prepared by melt blending in a Haake Minilab twin screw extruder following the optimal processing conditions previously studied. ${ }^{23}$ Subsequently, the materials were compression-moulded into $0.4 \mathrm{~mm}$ thick sheets in a Collin P200P press for mechanical testing, resulting in amorphous samples. The NR concentration in the blend was fixed at $10 \mathrm{wt} \%$, and the organoclay loadings were 1 and $3 \mathrm{wt} \%$.

\section{Structural characterisation}

The morphology of the samples was observed using a Philips XL30 environmental scanning electron microscope (ESEM) at $15 \mathrm{kV}$. All the samples were cryo-fractured after immersion in liquid nitrogen and the fracture surface was sputter coated with gold/palladium $(\mathrm{Au} / \mathrm{Pd} 80 / 20)$ in order to prevent electrical discharge during observation. The study of the dispersion of the nanoclays was carried out using transmission electron microscopy (TEM) (Philips Tecnai 20 microscope at an acceleration voltage of $200 \mathrm{kV}$ ). Ultra-thin sections of the samples were prepared by cryo-ultramicrotomy at $-140{ }^{\circ} \mathrm{C}$ (Leica EM UC6).

\section{Time resolved wide-angle X-ray scattering (WAXS) and small- angle X-ray scattering (SAXS) using synchrotron light}

Time-resolved WAXS and SAXS were performed at the Spanish beamline BM16-CRG of the European Synchrotron Facility
(ESRF) in Grenoble, France. ${ }^{24}$ The X-ray wavelength was $\lambda=$ $0.9795 \AA$ A. Data were collected using a MarCCD165 detector in $1024 \times 1024$ pixels resolution mode (effective pixel size of 158.7 $\mu \mathrm{m})$ at a sample to detector distance of $309.5 \mathrm{~mm}$ and $1397.4 \mathrm{~mm}$ for WAXS and SAXS, respectively. The experimental data were corrected for the X-ray absorption and background scattering. It should also be noted that the intensity was normalised by the sample thickness, $t$, at each step calculated from the BeerLambert law $I=I_{0} \exp (-\mu t)$. The absorption coefficient $\mu$ was determined for the undeformed samples and assumed to be constant during stretching. ${ }^{25}$

\section{Mechanical properties}

In situ WAXS and SAXS measurements were recorded simultaneously during longitudinal deformation of the samples by means of a Linkam tensile stage (Linkam Scientific Instruments Ltd.) at a speed of $5 \mathrm{~mm} \mathrm{~min}^{-1}$.

\section{Data analysis}

2D WAXS and SAXS patterns were analysed following different approaches in order to obtain as much structural information as possible about the stretching mechanism at the molecular level. From the 2D WAXS pattern (Fig. 1a) information about the orientation of polymer chains can be extracted. By enlarging the pattern at middle angles (Fig. 1b), the characteristic diffraction maximum of the nanoclay is clearly visible, confirming the intercalated structure of the nanocomposites (located at $q=1.68$ $\mathrm{nm}^{-1}$, i.e. $d=3.74 \mathrm{~nm}$ ), and the orientation of the nanoclay can be estimated. Further enlargement of the angular range provides access to smaller angles (Fig. 1c) where information about microvoids can be obtained.

\section{SAXS analysis}

Microvoid formation and orientation. 2D SAXS images (for example Fig. 1c) were integrated to obtain the scattering intensity $(I)$ as a function of the scattering vector $q,(q=4 \pi / \lambda \sin \theta$, where $2 \theta$ is the scattering angle). Moreover, we focused on a $q$-range from 0.1 to 1.2 $\mathrm{nm}^{-1}$ in order to study the microvoid formation and orientation. Thus, we calculated the total integrated intensity $I_{\text {tot }}$ defined by:

$$
I_{\mathrm{tot}}=\int_{0.1}^{1.2} I(q) \mathrm{d} q
$$

In order to estimate orientation fractions, 2D SAXS images were also divided in four quadrants, as pictured in Fig. $2 \mathrm{a} .^{26,27}$

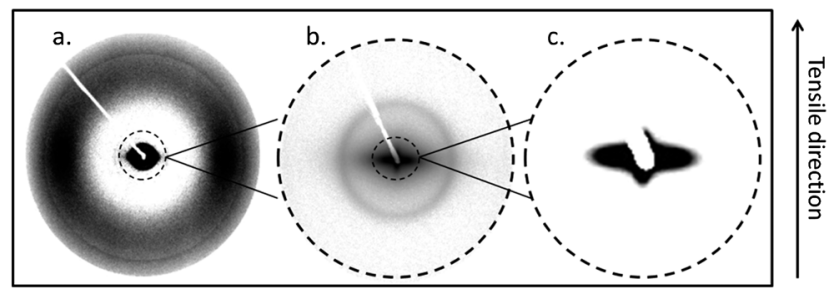

Fig. 1 Example of scattered intensity for an oriented sample (PLA/NR/ C15A $3 \mathrm{wt} \%$ at $50 \%$ strain) (a) WAXS 2D pattern: orientation of polymer chains, (b) orientation of nanoclays and (c) SAXS 2D pattern: microvoid formation and orientation. 
The meridional and equatorial scattered intensities were calculated as two total integrated intensities $I_{\text {mer }}$ and $I_{\text {eq }}$ over a $q$-range from 0.1 to $1.2 \mathrm{~nm}^{-1}$ and over azimuthal angles $(\varphi)$ from $-45^{\circ}$ to $45^{\circ}$ and $135^{\circ}$ to $225^{\circ}$ and from $45^{\circ}$ to $135^{\circ}$ and $225^{\circ}$ to $315^{\circ}$, respectively. According to Fig. $2 \mathrm{a}, I_{\mathrm{mer}}=I_{1}+I_{3}$ and $I_{\mathrm{eq}}=I_{2}+I_{4}$ and the oriented fraction is defined as $I_{\mathrm{or}}=I_{\mathrm{eq}} /\left(I_{\mathrm{eq}}+I_{\mathrm{mer}}\right)$.

Nanoclay orientation. Herman's orientation function can be used to estimate the degree of orientation of crystalline lamellae, or even polymer chains, from the resulting anisotropic response of scattering or spectroscopic tools. ${ }^{27,28}$ In our case, we used it to study the orientation of the nanoclays through the deformation induced anisotropy of the scattering associated with the average interlayer space of the nanoclays. The $q$-range for integration was taken from $q=1.4 \mathrm{~nm}^{-1}$ to $q=2.0 \mathrm{~nm}^{-1}$. Herman's orientation function $f_{2}$ is defined as:

$$
f_{2}=\frac{3\left\langle\cos ^{2} \varphi\right\rangle-1}{2}
$$

where

$$
\left\langle\cos ^{2} \varphi\right\rangle=\frac{\sum_{\varphi_{i}=0}^{90^{\circ}} I_{i} \sin \varphi_{i} \cos ^{2} \varphi_{i}}{\sum_{\varphi_{i}=0}^{90^{\circ}} I_{i} \sin \varphi_{i}}
$$

with $I_{i}$ the scattered intensity at the $i^{\text {th }}$ azimuthal angle $\varphi_{i}$ (see Fig. 2b). The initial and final angles in our case were chosen as $90^{\circ}$ and $180^{\circ}$ to avoid the shadow of the beam stop. In this case, $f_{2}$ is equal to 1 and -0.5 when the scattered intensity is concentrated along the meridian and the equator, respectively. The value of 0 is obtained for isotropic patterns.

\section{WAXS analysis}

Polymer chain orientation. Herman's orientation function was used in a similar way to determine the orientation of the disordered polymer chains during stretching through the amorphous halo observed in the 2D WAXS patterns (Fig. 1a). The $q$-range for integration was taken from $q=7.8 \mathrm{~nm}^{-1}$ to $14.2 \mathrm{~nm}^{-1}$.

\section{Results and discussion}

\section{Morphology of the materials}

Fig. 3 shows the SEM and TEM images of the blend and its nanocomposite at $3 \mathrm{wt} \%$ loading fraction of nanoparticles. The
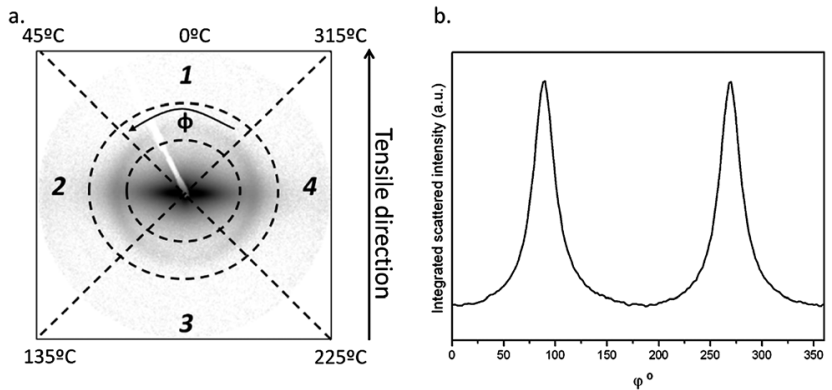

Fig. 2 (a) Example of SAXS analysis of 2D patterns (PLA/NR/C15A 3 $\mathrm{wt} \%$ at $100 \%$ strain). (b) Integrated intensity as a function of the azimuthal angle $\varphi$ in order to calculate Herman's orientation function.
PLA/NR blend presents phase separated morphology where the rubber particles form small dispersed droplets in the PLA matrix, with a low interfacial adhesion between the two polymers. The addition of the organoclay $\mathrm{C} 15 \mathrm{~A}$ to the blend decreases the rubber droplet size and leads to a more homogeneous morphology. As mentioned above, the organoclay is mainly located at the interface acting as an effective compatibiliser for the immiscible polymer blend. ${ }^{23}$

\section{Microstructure deformation mechanism as revealed by simultaneous WAXS, SAXS and stress-strain experiments}

The tensile behaviour of the materials is shown in Fig. 4 and 5a. PLA is a rigid and brittle polymer with a very low elongation at break. The addition of NR in the PLA matrix changes the brittle fracture of PLA to a ductile one with the formation and propagation of a neck while stretching. The stress reaches the maximum value at the yield point (strain 5\%) and then rapidly decreases, followed by a stable propagation up to fracture, reaching $200 \%$ elongation. Interestingly, a further increase of the elongation at break of up to $270 \%$ is obtained with the addition of $1 \mathrm{wt} \%$ of $\mathrm{C} 15 \mathrm{~A}$. However, this value drops to $120 \%$ when increasing the nanoclay concentration to $3 \mathrm{wt} \%$. We also observe that the addition of the nanoclays results in the disappearance of the neck and in the homogeneous deformation of the sample (see Fig. 4). ${ }^{23}$ These observed changes lead us to analyse the microstructure deformation mechanisms of PLA/NR/organoclay nanocomposites during uniaxial stretching by in situ synchrotron WAXS/SAXS techniques.

Simultaneous SAXS and WAXS patterns are presented in Fig. $5 \mathrm{~b}$ and $\mathrm{c}$.

SAXS patterns of stretched PLA are shown in Fig. 5b (first row). Fig. 6a shows the 1D-SAXS intensity of PLA at different elongations in the equatorial direction. PLA is a brittle material with a failure resulting from the formation of crazes, which are defined as microcracks bridged by small fibrils. ${ }^{29}$ Crazing is a commonly observed process of polymer deformation and is both a cause of failure and a mechanism of energy absorption,
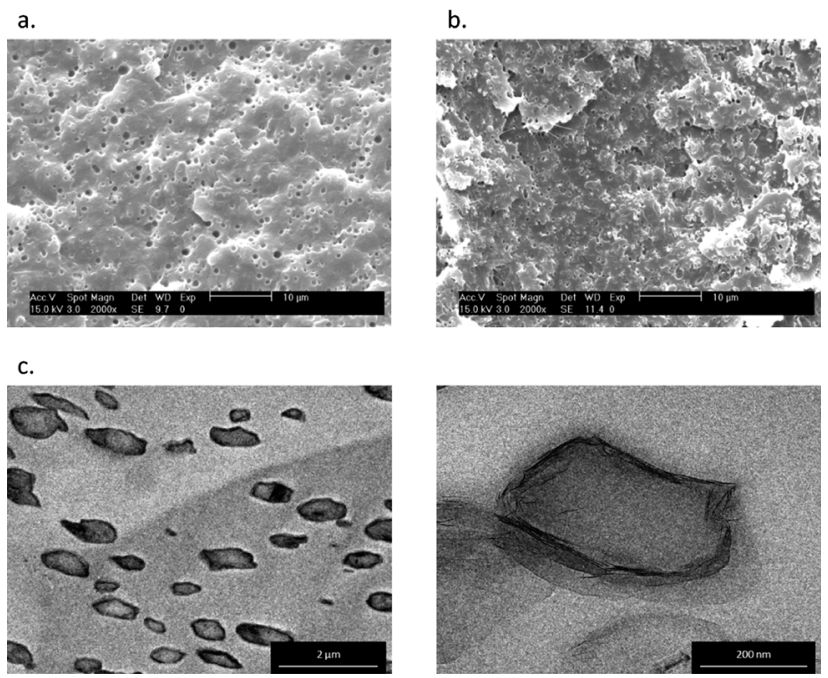

Fig. 3 Morphology of (a) PLA/NR blend, (b) PLA/NR/C15A 3 wt $\%$ nanocomposite, and (c) location of $\mathrm{C} 15 \mathrm{~A}$ in PLA/NR/C15A $3 \mathrm{wt} \%$ by TEM. 

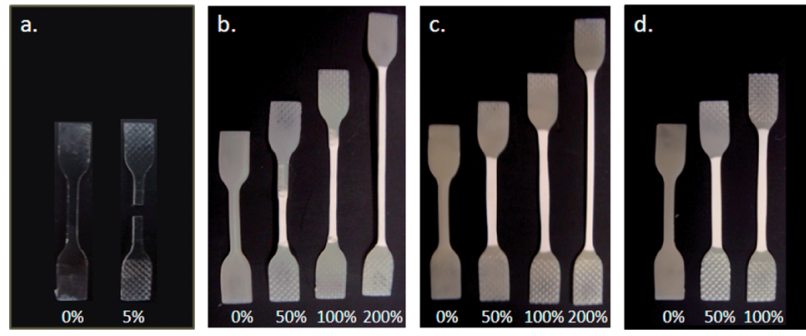

Fig. 4 Samples stretched at different elongations (a) PLA, (b) PLA/NR, (c) PLA/NR/C15A $1 \mathrm{wt} \%$, (d) PLA/NR/C15A $3 \mathrm{wt} \%$.
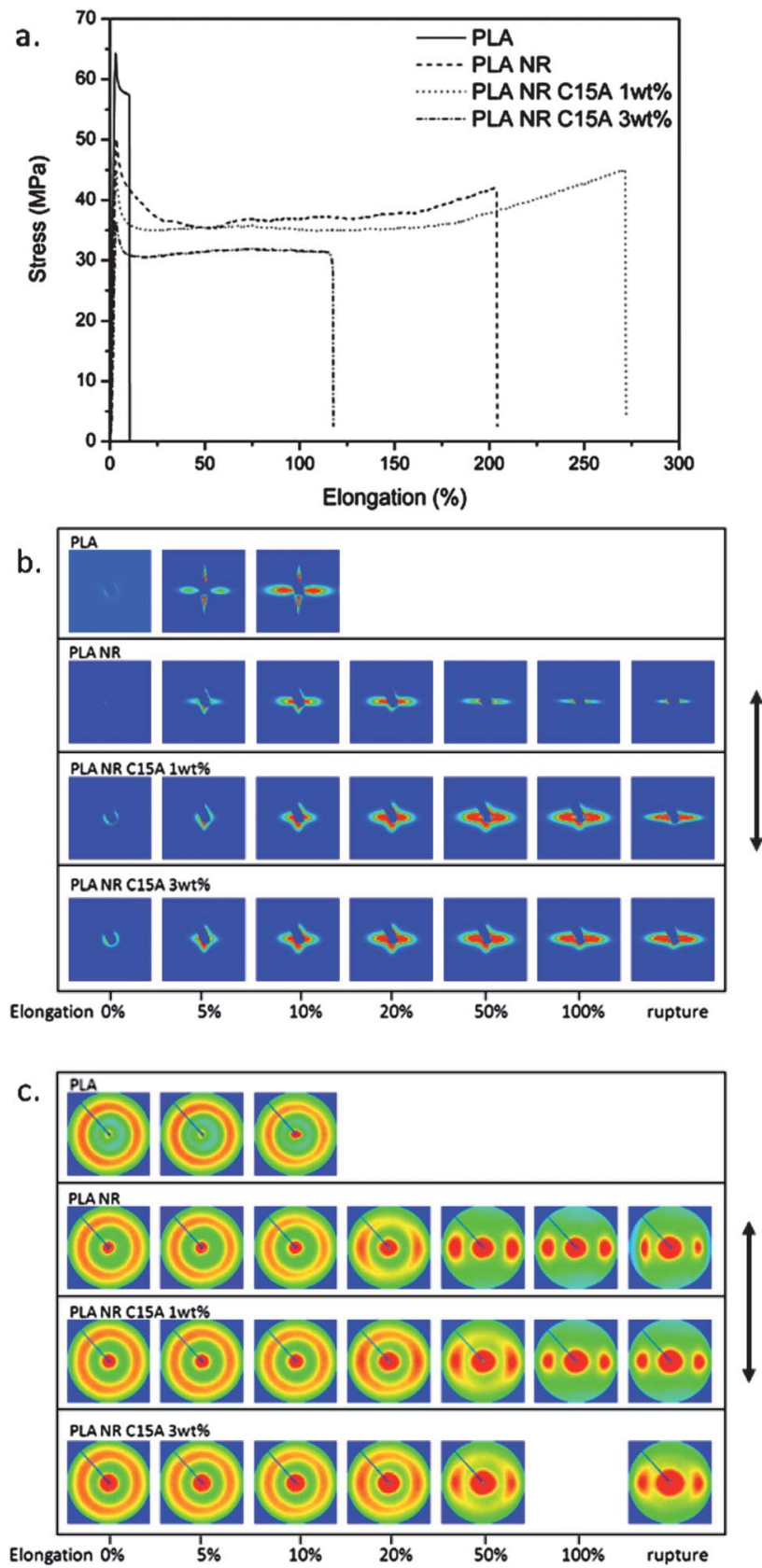

Fig. 5 (a) Mechanical properties of the PLA/NR blend and nanocomposites. The pictures present in situ simultaneous SAXS patterns (b) and WAXS patterns (c) at selected elongation values. The arrows indicate the stretching direction. depending on the craze formation and concentration. The generation of a high density of crazes can result in a tough system while few crazes can also develop into cracks, a precursor of failure. SAXS is an efficient technique to identify crazes because of their cross-like SAXS signature, resulting from the scattering of the craze fibrils in the equatorial direction and of the crack planes in the meridional direction. ${ }^{30}$

The maximum observed at $q=0.26 \mathrm{~nm}^{-1}$ for $10 \%$ strain in the scattered equatorial intensity suggests the development of crazes during stretching (Fig. 6a). In fact, it corresponds to the average correlation length between consecutive fibrils formed across the plane of craze. ${ }^{31}$

As previously shown, the addition of NR to the PLA matrix results in a large increase of the elongation at break. SAXS patterns of stretched PLA/NR blend are shown in Fig. 5b (second row). Prior to deformation, the PLA/NR blend presents a slight isotropic scattered intensity. At the yield point, the blend exhibits a sharp meridional intensity and equatorial strike close to the beam stop, which could indicate both the formation of crazes in the PLA matrix prior to the yield point and the appearance of the first microvoids at the interface between the PLA and NR phases due to a debonding process as previously proposed. ${ }^{22}$ As stretching progresses (up to $20 \%$ strain), the SAXS intensity increases in the equatorial direction (Fig. 6b), while no increase of signal is observed in the meridional one (Fig. 5b second row). This effect suggests that the formed microvoids are oriented parallel to the direction of the applied stress. This corresponds, at the macroscopic scale, to the whitening of the blend as a consequence of microvoid formation and also to the formation of a neck due to the microscopic
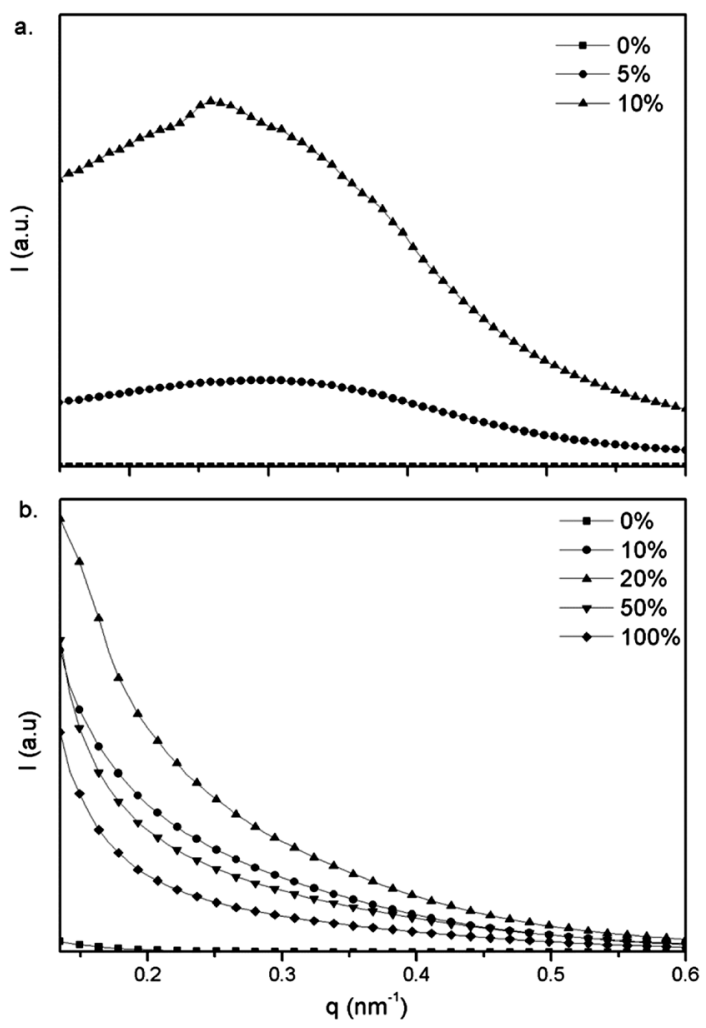

Fig. 6 1D-SAXS intensity (linear scale) integrated in the equatorial region of (a) PLA and (b) PLA/NR blend. 
localisation of the stress at the yield point. When the specimens are further stretched, no significant changes in the SAXS patterns are observed due to the propagation of the neck through the sample. Neither a peak nor a shoulder are observed in the 1D-scattered intensity in the equatorial direction (Fig. 6b). However, we observe a decrease of the intensity at low $q$-values in the equatorial direction.

The absence of an equatorial intensity maximum for PLA/NR blend (Fig. 6b) in comparison with its presence for PLA (Fig. 6a) is a clear indication of changes in the mechanisms of deformation. Previous scattering studies on poly(styrene)/elastomer blends by Magalhaes and Borggreve reported a similar effect which was attributed to non-crazing processes and to the formation of irregular cavities. ${ }^{31}$ Consequently, in our case we can infer that crazing is not the main deformation process for the PLA/NR blend in contrast to PLA. Cavitation and debonding are the two other traditionally proposed deformation mechanisms for thermoplastic/elastomer blends, depending on the phase morphology and adhesion. ${ }^{10}$ Since we showed in a previous study that adhesion between PLA and NR is poor, then debonding should prevail as the main deformation mechanism here. $^{22}$

If we now focus on the SAXS patterns for the nanocomposites (Fig. 5b, third and fourth row), some important changes can be observed in comparison to the PLA/NR blend. First of all, we observe an orientation of the scattering intensity in the meridional direction at low elongations, which means that the microvoids are oriented perpendicular to the direction of the applied stress. After $10 \%$ strain, a cross-like pattern appears. Subsequently for higher strain, the equatorial intensity becomes even stronger and the signal in the meridional direction decreases. The 1D-SAXS equatorial intensity for the PLA/NR/C15A for $1 \mathrm{wt} \%$ and $3 \mathrm{wt} \%$ of nanoclay are shown in Fig. $7 \mathrm{a}$ and $\mathrm{b}$. For $1 \mathrm{wt} \%$ of nanoclays, a shoulder appears at low angles for strains higher than $20 \%$ which evolves to a maximum for strains between $50 \%$ and $100 \%$. This maximum then tends to disappear progressively for strains higher than $100 \%$ until sample failure. Similar observations are made for $3 \mathrm{wt} \%$ fraction loading of nanoclays.

The appearance of this maximum could result from the scattering of the craze fibrils and indicates that the addition of the nanoclays tends to suppress the debonding process of rubber droplets in favour of craze formation. Moreover, additional experiments demonstrated that craze formation is inhibited in favour of cavitation in the PLA/C15A nanocomposite during stretching (data not shown), indicating that the reason for crazing mechanisms in PLA/NR/C15A bionanocomposites is the combined effect of NR and C15A. Thus, our results suggest that the nanoclay located at the PLA-NR interface (Fig. 3) acts as a compatibilising agent hindering the formation of voids between the two phases. The further disappearance of the maximum for strains higher than $100 \%$ in the case with $1 \mathrm{wt} \%$ of clay loading could correspond to the transformation of the crazes into larger voids.

Fig. 8 represents the evolution of the oriented fraction of microvoids of the PLA/NR blend and nanocomposites as a function of the applied strain. As observed for the PLA/NR blend, a rapid initial orientation of the voids takes place until a strain of about $30 \%$ is reached. Subsequent strain has no further effect. In contrast, for PLA/NR/C15A nanocomposites, the
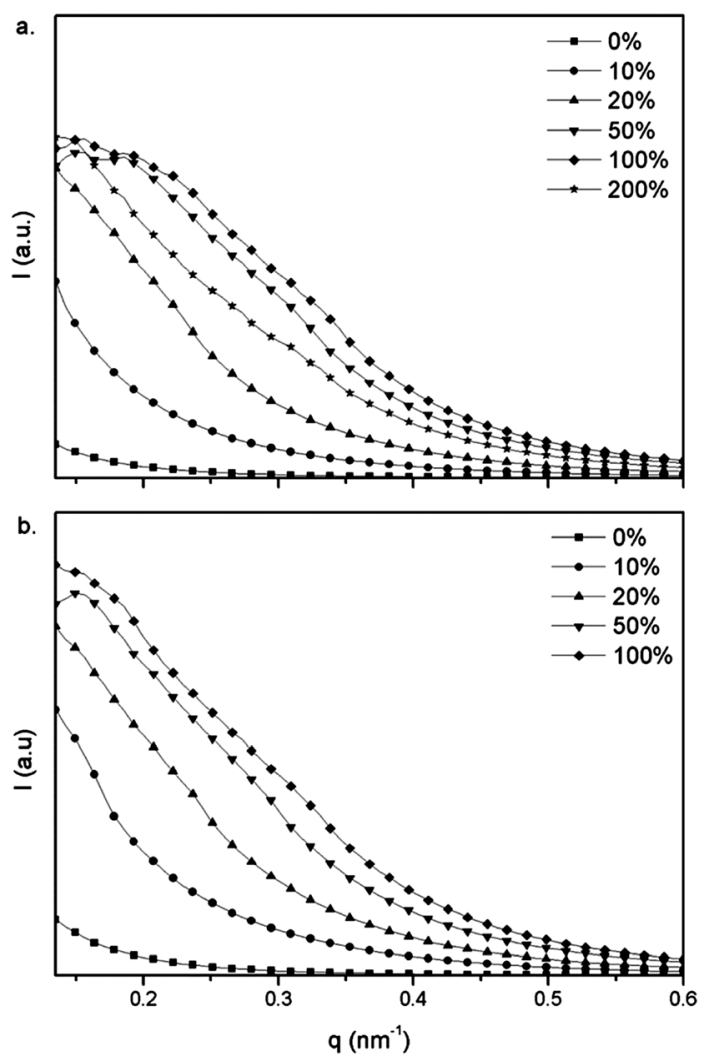

Fig. 7 1D-SAXS intensity (linear scale) integrated in the equatorial region for (a) PLA/NR/C15A $1 \mathrm{wt} \%$ and (b) PLA/NR/C15A $3 \mathrm{wt} \%$.

oriented fraction of voids progressively increases with strain until break. No effect is observed on C15A concentration. These results are in agreement with the macroscopic observation of a more homogenous deformation of nanocomposites than that of the blend (see Fig. 4).

Fig. 9 shows the evolution of the total integrated intensity (integrated from $q=0.1$ to $1.2 \mathrm{~nm}^{-1}$ ) as a function of elongation. In a first approach, we can attribute the increase of the intensity to the void formation since the scattered intensity is determined by the differences of electron density. In fact, X-ray scattering

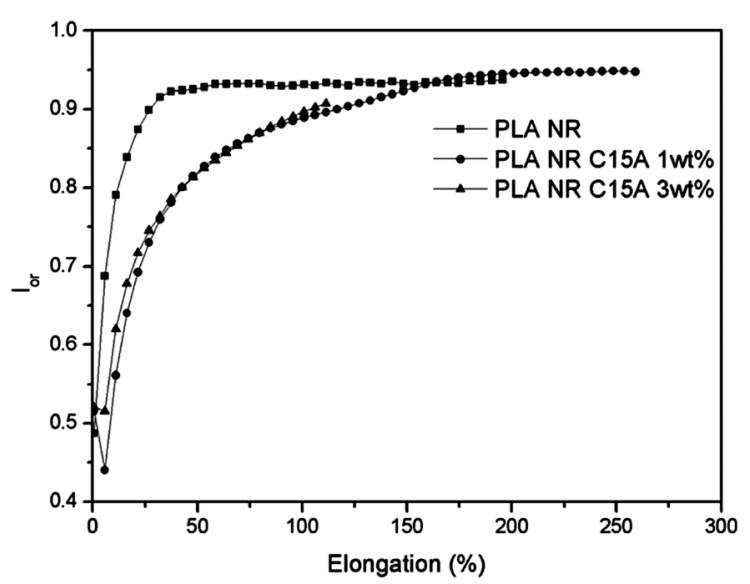

Fig. 8 Oriented fraction of microvoids of the PLA/NR blend and nanocomposites. 


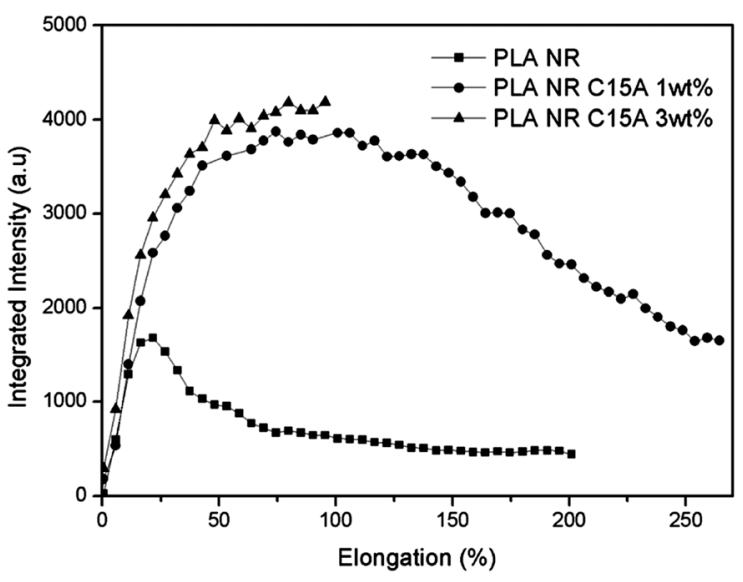

Fig. 9 Variation of total integrated intensity over $q=0.1$ to $1.2 \mathrm{~nm}^{-1}$.

intensity is proportional to the square of the electron density contrast. If we consider that the void electron density is about two orders of magnitude lower than those of the polymers, then the observed increase of the total integrated intensity can be assigned to an increase of microvoid concentration..$^{32}$ In the case of the PLA/NR blend, the integrated intensity increases up to $20 \%$ and then decreases, reaching a stable plateau value corresponding to the propagation of the neck. Since the intensity is normalised by the sample thickness, the reduction of sample volume could not be the explanation for this decrease of intensity. Therefore, we propose that the voids reach a size as large as to be beyond the resolution. The total integrated intensity of the nanocomposites is significantly higher than that of the PLA/NR blend. Moreover, a slight increase is observed with the concentration of nanoclays. A maximum is reached at $100 \%$ strain for 1 $\mathrm{wt} \%$ clay loading, meanwhile the sample breaks before showing any maximum for $3 \mathrm{wt} \%$ loading.

From the scattering analysis of PLA/NR blend, we can conclude that the formation (Fig. 9) and orientation of microvoids (Fig. 8) occur at the first stage of sample deformation up to about $30 \%$ strain. Thus, we can infer that at this initial stage of deformation, the stress is concentrated around the NR droplets and that debonding gives rise to the formation of the first microvoids. This corresponds at a macroscopic scale to the formation of the neck. As deformation increases, the voids stabilise, allowing the propagation of the neck along the sample.

Furthermore, the strong increase of total integrated intensity of the nanocomposites when compared to that of the PLA/NR blend (Fig. 9) clearly demonstrates that the nanoclay platelets could act as preferential sites to induce craze formation. For $1 \mathrm{wt}$ $\%$ of $\mathrm{C} 15 \mathrm{~A}$, the concentration and density of crazes increase and become stable until $100 \%$ strain. Beyond this point, the intensity decreases probably due to the progressive rupture of polymer fibrils of the crazes and to their disappearance to form larger voids or "cracks" which keep growing and orienting in the tensile direction. For $3 \mathrm{wt} \%$ of clays, the progressive transformation of crazes to cracks results in the mechanical failure of the sample, explaining the reduction of elongation at break with the clay concentration. Thus, our results indicate that an optimal number of crazes able to become stable are generated for $1 \mathrm{wt} \%$ nanoclay.
Nanoclay orientation as revealed by intermediate angle X-ray scattering

The orientation of the nanoclays was analysed according to Herman's orientation function $f_{2}$. SAXS patterns and $f_{2}$ are presented as a function of the elongation in Fig. 10. Close inspection of X-ray patterns (Fig. 10a) and Herman's orientation function indicate that the nanoparticles progressively orient themselves in the direction of the applied stress, verifying also the orientation of the PLA/NR interface.

Further observation of these SAXS patterns reveals that the diffraction maximum of the nanoclays not only becomes oriented but also broadens with stretching. This effect can be visualised in Fig. 11 where we represent the equatorial intensity for the PLA/NR/C15A $3 \mathrm{wt} \%$ nanocomposite. Two reflections with a maximum intensity at $q=1.68 \mathrm{~nm}^{-1}$ ( $1^{\text {st }}$ order), and $3.4 \mathrm{~nm}^{-1}$ $\left(2^{\text {nd }}\right.$ order) corresponding to the layered structure of the nanoclays $(d=3.74 \mathrm{~nm})$ are observed and do not suffer any modification upon stretching. In contrast, a clear broadening of the main peak and a disappearance of the $2^{\text {nd }}$ order one are clearly evidenced, suggesting that a loss of the nanoclays crystalline perfection occurs during stretching without delamination of the clays. ${ }^{33}$

\section{Orientation of the polymer chains as revealed by WAXS}

Herman's orientation function is calculated from the scattering patterns in the WAXS angular region (Fig. 5c). In this range $\left(q\right.$-range from $7.8 \mathrm{~nm}^{-1}$ to $\left.14.2 \mathrm{~nm}^{-1}\right)$ the amorphous halo of both PLA and natural rubber can be detected. The analysis
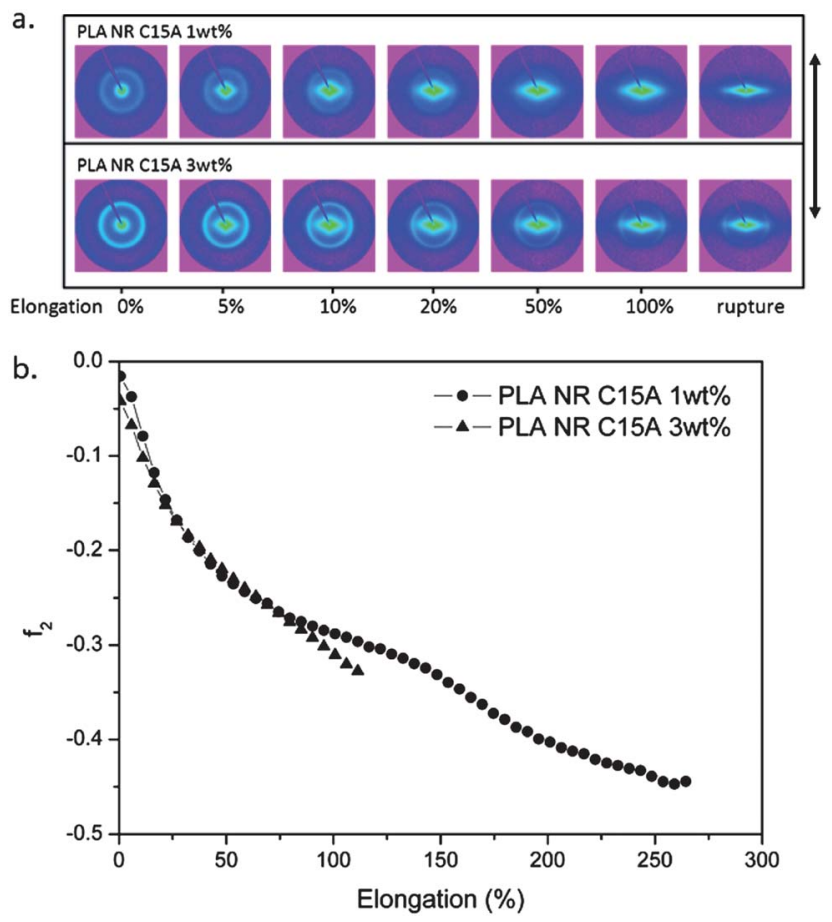

Fig. 10 (a) X-ray scattering patterns at intermediate angles for selected elongations and (b) Herman's orientation function variation with the elongation for PLA/NR/C15A nanocomposites. The arrows indicate the stretching direction. 


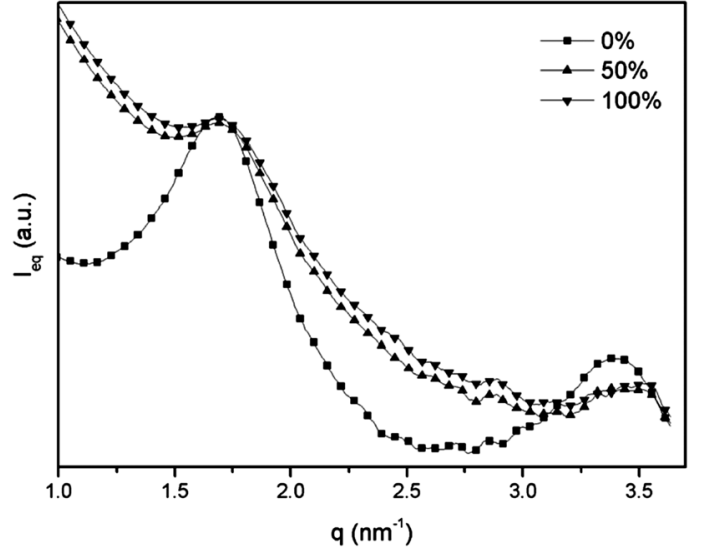

Fig. 11 Equatorial X-ray intensity (logarithmic scale) at intermediate angles for PLA/NR/C15A $3 \mathrm{wt} \%$ nanocomposites.

of this halo is used to evaluate the polymer chain orientation during deformation (Fig. 12). Since the observed halo includes both the PLA and the NR contributions, it is not possible to isolate them from the WAXS pattern.

For the PLA/NR blend, we observe a strong orientation development from 0 to $50 \%$. The formation and orientation of voids also take place at this elongation range. In fact, these two phenomena permit to lower the yield strength and facilitate the orientation of polymer chains as observed in Fig. 12. The elongation of the PLA matrix can occur in PLA/NR facilitated by the dissipation of energy by the void formation, giving rise to a ductile fracture mechanism. For elongations higher than $50 \%$ no further orientation is observed. However, a slight change occurs after 150\% elongation. This change could correspond to the end of the neck propagation. After that, further orientation of the voids (Fig. 8) and of the polymer chains (Fig. 12) takes place, resulting in strain hardening by the extension of the molecular network until the sample breaks. ${ }^{34}$

A similar qualitative effect is observed for the nanocomposites with a shift towards higher elongations. As we previously discussed, the concentration of crazes increases with the nanoclay concentration, resulting in a higher dissipation of the applied energy through the formation and growth of voids. Therefore,

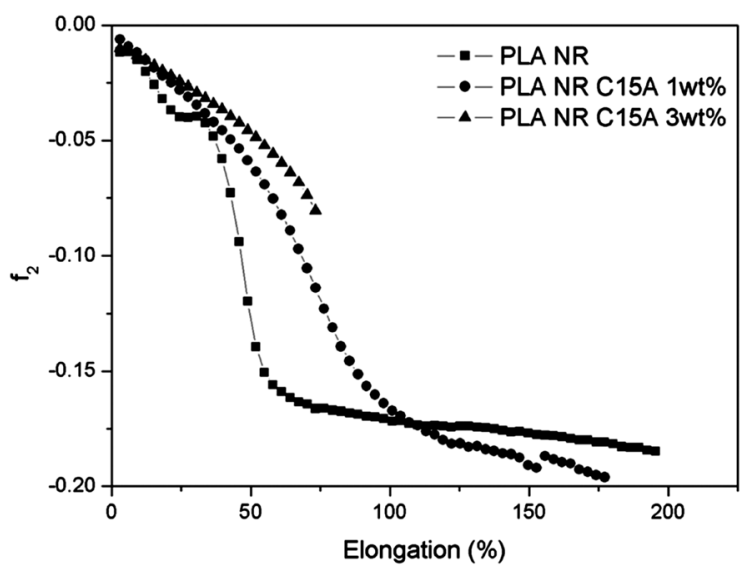

Fig. 12 Herman's orientation function versus elongation for the PLA/ NR blend and its nanocomposites (from WAXS patterns). the energy attributed to the plastic deformation of the polymeric matrix materials is lowered, explaining the decrease of the polymer chain orientation when increasing the nanoclay concentration (Fig. 12). Similar results have been obtained by $\mathrm{Na}$ et al..$^{34}$ These observations also explain the reduction of the flow stress with the filler concentration in the mechanical properties of the materials.

\section{Conclusions}

The influence of an organoclay addition to a PLA/NR blend on the deformation micromechanisms has been studied through in situ SAXS and WAXS measurements under tensile conditions and allowed us to understand how the bionanocomposite can be more ductile than the simple PLA/NR blend. The deformation mechanisms of each material were analysed and can be itemised as follows: (1) PLA is a brittle material with a failure through the formation of crazes. (2) PLA/NR is a ductile blend in which debonding associated with the formation of voids occurs when stretching. (3) PLA/NR/C15A bionanocomposites upon deformation exhibit craze formation since the location of the nanoclays at the PLA/NR interface hinders the formation of voids between the two phases. The nanoclays act as preferential sites for the craze formation and their number increases with the filler concentration. However, while the PLA matrix cannot stand formation of crazes or cavities for large elongations without mechanical failure, the presence of organoclays favours the generation of a large number of crazes in PLA/NR blends that are able to develop higher deformations. This mechanism allows, under elongation, a progressive orientation of polymer chains, nanoparticles and interfaces. Moreover, it appears that $1 \mathrm{wt} \%$ of nanoclay is the optimum concentration in our blend regarding the mechanical properties. A higher organoclay concentration precludes craze development and their transformation into cracks results in the sample mechanical failure. Thus, a clear synergetic effect is revealed in the mechanical reinforcement mechanism of the PLA matrix obtained with the addition of NR and the right amount of nanofillers. Moreover, the proposed mechanisms could be applied to other polymer blend nanocomposites in which the nanofiller is located at the interface between the immiscible polymers.

\section{Acknowledgements}

The authors thank the ESRF for beam-time and François Fauth for technical assistance during experiment. The work was supported by the Spanish Ministry of Science and Innovation (MICINN) under projects MAT2010-18749, MAT2009-07789 and MAT2008-03232. NB thanks the CSIC for a JAE-Pre grant. AS acknowledges the financial support through the project FIS 2010-15502 (Dirección general de Investigación, Spain).

\section{Notes and references}

1 A. Sodergard and M. Stolt, Prog. Polym. Sci., 2002, 27, 1123-1163.

2 R. C. Thomson, M. C. Wake, M. J. Yaszemski and A. G. Mikos, $A d v$. Polym. Sci., 1995, 122, 245-274.

3 H. D. Kim, E. H. Bae, I. C. Kwon, R. R. Pal, J. D. Nam and D. S. Lee, Biomaterials, 2004, 25, 2319-2329.

4 I. Armentano, M. Dottori, E. Fortunati, S. Mattioli and J. M. Kenny, Polym. Degrad. Stab., 2010, 95, 2126-2146. 
5 R. Bhardwaj and A. K. Mohanty, Biomacromolecules, 2007, 8, 24762484.

6 L. Jiang, M. P. Wolcott and J. W. Zhang, Biomacromolecules, 2006, 7, 199-207.

7 L. A. Utracki, Polymer Blends Handbook, Kluwer academic publishers, 2002.

8 F. S. Bates, Science, 1991, 251, 898-905.

9 S. H. Wu, Polymer, 1985, 26, 1855-1863.

10 G. M. Kim and G. H. Michler, Polymer, 1998, 39, 5689-5697.

11 G. M. Kim and G. H. Michler, Polymer, 1998, 39, 5699-5703.

12 J. S. Hong, Y. K. Kim, K. H. Ahn, S. J. Lee and C. Kim, Rheol. Acta, 2007, 46, 469-478.

13 L. T. Vo and E. P. Giannelis, Macromolecules, 2007, 40, 82718276.

14 K. Y. Wang, Y. M. Chen and Y. Zhang, Polymer, 2008, 49, 33013309.

15 F. Fenouillot, P. Cassagnau and J. C. Majeste, Polymer, 2009, 50, $1333-1350$.

16 S. M. L. Silva, M. A. Lopez-Manchado and M. Arroyo, J. Nanosci. Nanotechnol., 2007, 7, 4456-4464.

17 A. Dasari, Z. Z. Yu, M. S. Yang, Q. X. Zhang, X. L. Xie and Y. W. Mai, Compos. Sci. Technol., 2006, 66, 3097-3114.

18 A. Nogales, I. Sics, T. A. Ezquerra, Z. Denchev, F. J. B. Calleja and B. S. Hsiao, Macromolecules, 2003, 36, 4827-4832.

19 J. J. Hernandez, M. C. Garcia-Gutierrez, A. Nogales, D. R. Rueda, A. Sanz, I. Sics, B. S. Hsiao, Z. Roslaniec, G. Broza and T. A. Ezquerra, Polymer, 2007, 48, 3286-3293.

20 R. M. A. l'Abee, M. van Duin, A. B. Spoelstra and J. G. P. Goossens, Soft Matter, 2010, 6, 1758-1768.
21 C. B. He, A. M. Donald and M. F. Butler, Macromolecules, 1998, 31, $158-164$.

22 N. Bitinis, R. Verdejo, P. Cassagnau and M. Lopez-Manchado, Mater. Chem. Phys., 2011, 129, 823-831.

23 N. Bitinis, R. Verdejo, E. M. Maya, E. Espuche, P. Cassagnau and M. A. Lopez-Manchado, Compos. Sci. Technol., 2012, 72, 305-313.

24 D. R. Rueda, M. C. Garcia-Gutierrez, A. Nogales, M. J. Capitan, T. A. Ezquerra, A. Labrador, E. Fraga, D. Beltran, J. Juanhuix, J. F. Herranz and J. Bordas, Rev. Sci. Instrum., 2006, 77, 033904033905.

25 X. Li, K. Schneider, B. Kretzschmar and M. Stamm, Macromolecules, 2008, 41, 4371-4379.

26 L. B. Li and W. H. de Jeu, Macromolecules, 2004, 37, 5646-5652.

27 M. C. Garcia-Gutierrez, J. J. Hernandez, A. Nogales, P. Pantine, D. R. Rueda and T. A. Ezquerra, Macromolecules, 2008, 41, 844-851.

28 G. Gorrasi, R. Di Lieto, G. Patimo, S. De Pasquale and A. Sorrentino, Polymer, 2011, 52, 1124-1132.

29 A. C. Renouf-Glauser, J. Rose, D. F. Farrar and R. E. Cameron, Biomaterials, 2005, 26, 5771-5782.

30 C. B. He, T. X. Liu, W. C. Tjiu, H. J. Sue and A. F. Yee, Macromolecules, 2008, 41, 193-202.

31 A. M. L. Magalhaes and R. J. M. Borggreve, Macromolecules, 1995, 28, 5841-5851.

32 Y. Ijichi, T. Kojima, Y. Suzuki, T. Nishio, M. Kakugo and Y. Amemiya, Macromolecules, 1993, 26, 829-835.

33 A. Sanz, D. R. Rueda, A. Nogales and T. A. Ezquerra, Journal of Nanostructured Polymers and Nanocomposites, 2011, 7, 10-17.

34 B. Na, W. Xu, R. Lv, N. Tian, Z. Li, R. Su and Q. Fu, J. Polym. Sci., Part B: Polym. Phys., 2010, 48, 514-519. 